\title{
The ATLAS New Small Wheel Upgrade Project
}

\author{
Konstantinos Ntekas* \\ on behalf of the ATLAS Muon Collaboration \\ National Technical University of Athens, Brookhaven National Laboratory \\ E-mail: konstantinos.ntekas@cern.ch
}

\begin{abstract}
The upgrade of the Large Hadron Collider (LHC) at CERN foresees a luminosity increase by a factor 5. To cope with the corresponding rate increase, the ATLAS detector needs to be upgraded. The upgrade will proceed in two steps: Phase-I in the LHC shutdown 2018/19 and Phase-II in 2023-25. The largest of the ATLAS Phase-I upgrades concerns the replacement of the first muon station in the high-rapidity region, the so called New Small Wheel (NSW). It employs eight layers of Micromegas detectors (MM) and eight layers of small-strip Thin Gap Chambers (sTGC). This configuration copes with the highest rates expected in Phase-II and enhances the performance of the forward muon system by adding trigger functionality to the first muon station. We describe the limitations of the present muon detector and the expected improvements, the requirements for the NSW along with the detector layout, including trigger \& readout electronics based on a newly designed front end ASIC. We conclude with an update of the status of the project and the steps towards a complete operational system, ready to be installed in ATLAS in 2018/19.
\end{abstract}

Technology and Instrumentation in Particle Physics 2014,

2-6 June, 2014

Amsterdam, the Netherlands

\footnotetext{
* Speaker.
} 


\section{ATLAS Muon Spectrometer Upgrade - Motivation for New Small Wheels}

The ATLAS upgrade is motivated primarily by the high background radiation that is expected at $\mathscr{L}=5 \times 10^{34} \mathrm{~cm}^{-2} \mathrm{~s}^{-1}$, after the High Luminosity LHC upgrade. This will lead to a particle rate up to $15 \mathrm{kHz} / \mathrm{cm}^{2}$, in the Small Wheel region (SW) (Figure 1 left), which the present SW detectors (MDT, CSC \& TGC) cannot handle efficiently. Also, Endcap-Muon (EM) triggers are dominated by fakes $(>90 \%)$ (Figure 1 right) due to background, low energy particles generated in the material located between the SW and the EM station hitting the EM at an angle similar to that of real high $p_{T}$ muons.
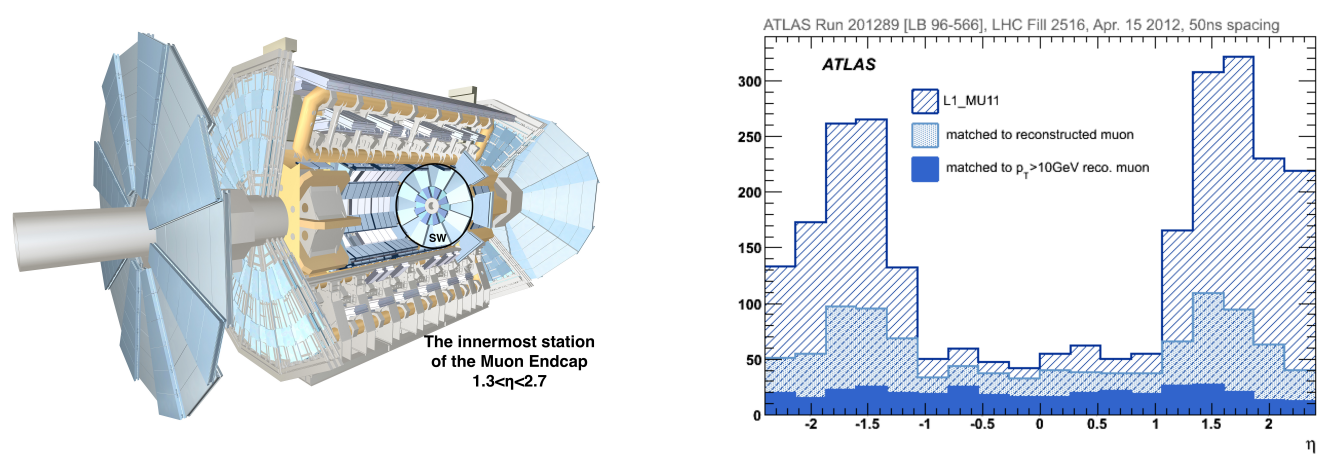

Figure 1: Left: The Muon Spectrometer of the ATLAS Experiment. Right: $\eta$ distribution of Level-1 muon signal $\left(p_{T}>10 \mathrm{GeV}\right)$ with the distribution of the subset with matched muon candidate to an offline well reconstructed muon (combined inner detector and muon spectrometer track with $p_{T}>3 \mathrm{GeV}$ ), and offline reconstructed muons with $p_{T}>10 \mathrm{GeV}$.

Raising of the muon trigger threshold to reduce the level-1 trigger rate results in a significant loss of physics acceptance. A New Small Wheel system (NSW) [1], with high rate capability will be developed, that will be included in the level-1 trigger. This will minimize the fake triggers by reconstructing high quality $\left(\sigma_{\theta} \sim 1 \mathrm{mrad}\right)$ Interaction Point (IP) pointing segments (Figure 2), providing also efficient \& precise tracking for the expected rate of $15 \mathrm{kHz} / \mathrm{cm}^{2}$.
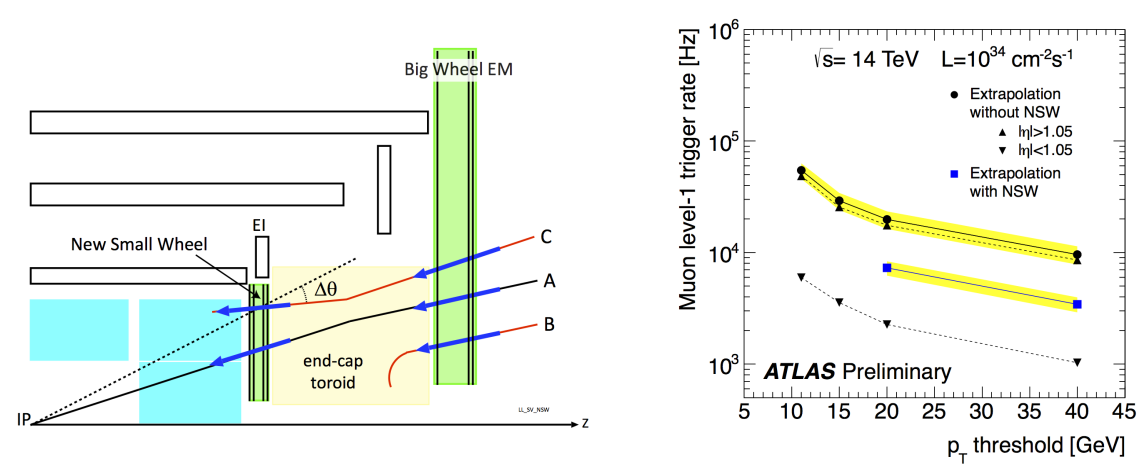

Figure 2: Left: The existing Big Wheel (BW) trigger accepts all three tracks shown. With the NSW enhancement of the EM trigger only track 'A', which is confirmed by both the BW and the NSW, will be accepted. Right: Estimation of ATLAS muon level-1 trigger rate extrapolated for $p p$ collisions at $\sqrt{ } s=$ $14 \mathrm{TeV}$ with instantaneous luminosity of $\mathscr{L}=10^{34} \mathrm{~cm}^{-2} \mathrm{~s}^{-1}$, shown as a function of $p_{T}$ threshold. The extrapolation with the NSW is also shown. 


\section{Detector Layout}

The NSW (Figure 3 left) will be composed of sTGC \& Micromegas (MM) detectors. Both technologies will provide tracking and triggering information, resulting into a fully redundant NSW system. Each of the two wheels will consist of sixteen sectors (8 large, 8 small) with four wedges in each sector in sTGC-MM-MM-sTGC configuration as shown in Figure 3 right. Each wedge combines four detecting layers with half of the MM layers in a stereo angle layout for $2^{\text {nd }}$ coordinate measurement.
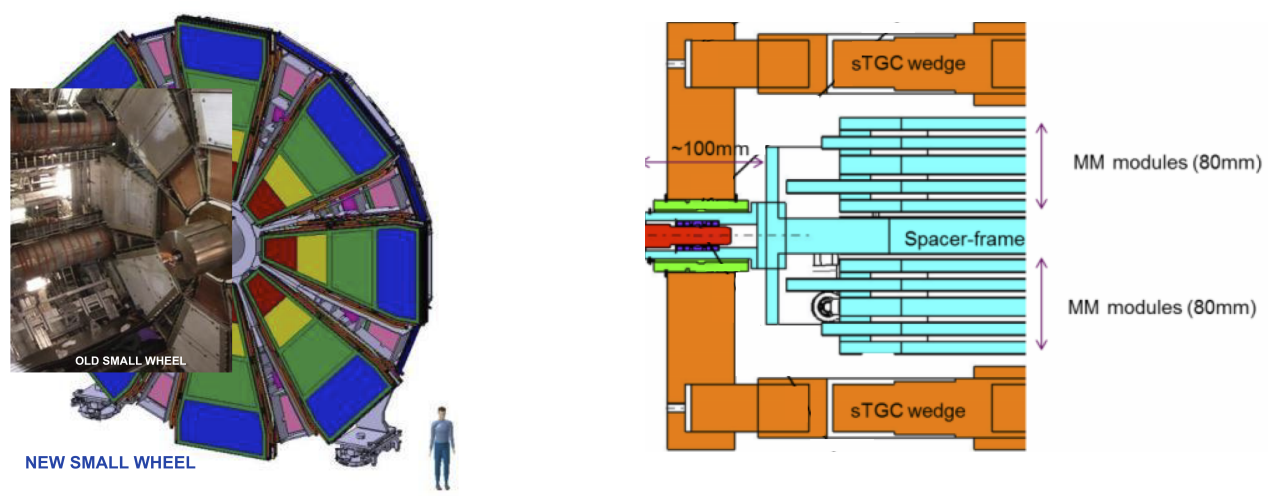

Figure 3: Left: Sketch of the NSW structure compared to the current SW. Right: Cross section of a NSW sector integrated with its mechanical support structure.

The sTGC will be the primary trigger detector featuring bunch crossing (BC) identification capability (Figure 4 left) and angular resolution for online reconstructed segments better than $1 \mathrm{mrad}$ [2]. The detector is also characterized by good spatial resolution for the offline tracking using the precision coordinate (readout strips) and it is based on proven TGC technology, adding also pads for muon track identification and readout region definition [3].
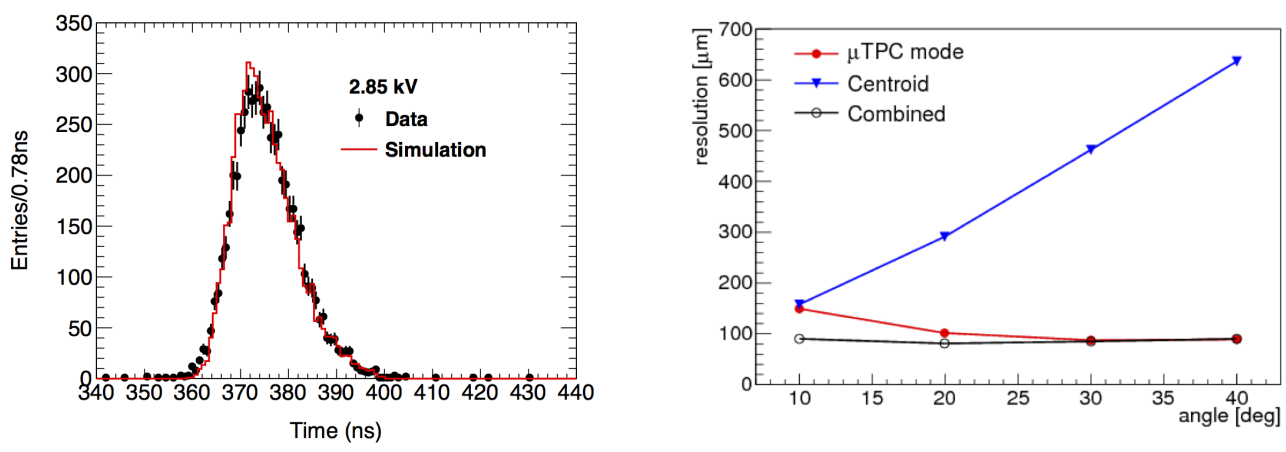

Figure 4: Left: Comparison of a simulated time spectrum with experimental data for muons transversing normally an sTGC detector (95\% of the events are contained within a $25 \mathrm{~ns}$ time window). Right: MM detector spatial resolution along different values of incident angle, with charge centroid method (blue triangles), using the MM as a TPC (red circles) and combining the two methods (black circles).

The newly developed MM detector [4] will be the primary precision tracker with spatial resolution better than $100 \mu \mathrm{m}$ independent of track incident angle (Figure 4 right). Also, the hit with the 
earliest arrival time every BC will be used as a trigger primitive [5]. This novel technology is characterized by excellent high rate capability [6] due to thin amplification gap and small space charge effects.

\section{Readout Electronics}

The newly developed VMM chip [7] is a 64-channel front-end ASIC, common for both technologies that will be used to readout the $\sim 2.5 \mathrm{M}$ channels of the NSW. It can provide charge measurement up to $2 \mathrm{pC}$ with $1 \mathrm{fC}$ uncertainty and time measurement with accuracy better than $1 \mathrm{~ns}$ at $100 \mathrm{~ns}$, featuring also an adjustable gain of $(0.5-16 \mathrm{mV} / \mathrm{fC})$. The $2^{\text {nd }}$ generation of the chip will be produced and tested in 2014, while the full production of the final electronics is scheduled for 2016, according to the NSW time-plan shown in Figure 5.

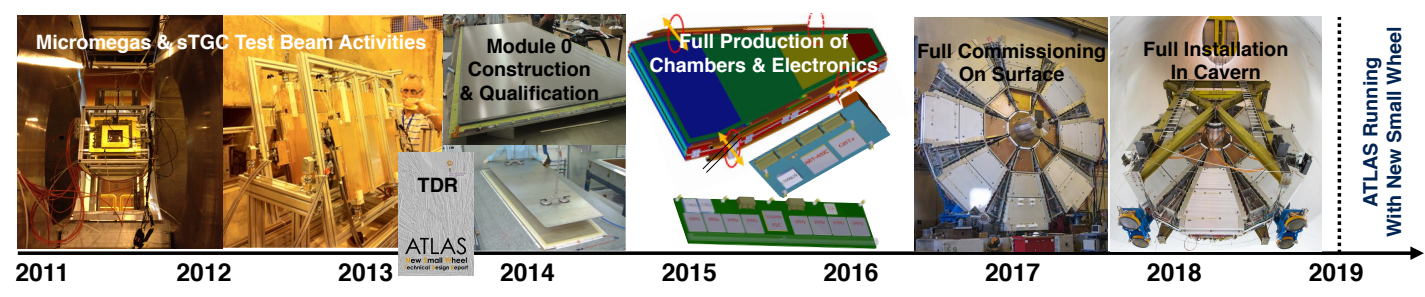

Figure 5: The NSW Project Timeline.

\section{Acknowledgments}

The present work was co-funded by the European Union (European Social Fund ESF) and Greek national funds through the Operational Program "Education and Lifelong Learning" of the National Strategic Reference Framework (NSRF) 2007-1013. ARISTEIA-1893-ATLAS MICROMEGAS.

\section{References}

[1] ATLAS Collaboration, NSW TDR, CERN-LHCC-2013-006, ATLAS-TDR-020 (2013).

[2] Y. Benhammou et al., Test of spatial resolution and trigger efficiency of a combined Thin Gap and Fast Drift Tube Chambers for high-luminosity LHC upgrades, IEEE Nucl.Sci.Symp.Conf.Rec. 2011 (2011) 1761-1766.

[3] V. Smakhtin et al., Thin Gap Chamber upgrade for SLHC: Position resolution in a test beam, Nucl. Instr. Meth. A 598 (2009) 196-200.

[4] T. Alexopoulos et al., A spark-resistant bulk-micromegas chamber for high-rate applications, Nucl. Instr. Meth. A 640 (2011) 110-118.

[5] G. Iakovidis, The Micromegas project for the ATLAS upgrade, 2013 JINST 8 C12007.

[6] Y. Kataoka et al., Performance studies of a micromegas chamber in the ATLAS environment, 2014 JINST 9 C03016.

[7] G. De Geronimo, et al., VMM1 - an ASIC for micropattern detectors, IEEE TRANSACTIONS ON NUCLEAR SCIENCE, VOL. 60, NO. 3, JUNE 2013. 